\title{
Electromagnetic and gravitomagnetic structure and radii of nucleons
}

\author{
Oleg Selyugin ${ }^{1,3, *}$ \\ ${ }^{1}$ BLTPh, JINR, Dubna, Russia
}

\begin{abstract}
Taking into account the PDFs, obtained by different Collaborations, the momentum transfer dependence of GPDs of the nucleons is obtained. The calculated electromagnetic and gravitomagnetic form factors of nucleons are used for the description of different form factors and the nucleons elastic scattering in a wide energy and momentum transfer region with a minimum number of fitting parameters. The electromagnetic and gravitomagnetic radii of the nucleon are calculated using the obtained momentum transfer dependence of GPDs with different forms of PDFs obtained by different Collaborations. The comparison of the calculations, taking into account the PDFs obtained by different Collaborations, of mean square electromagnetic and gravitomagnetic radii of nucleons is made.
\end{abstract}

\section{Introduction}

One of the most important tasks of the Standard model is the research of the structure of "elementary" particles. The main integral properties of particles are reflected in their radii. This is tightly connected with the particle form factors. This is primarily due to the electromagnetic structure of the nucleon which can be obtained from the electron-hadron elastic scattering. In the Born approximation, the Feynman amplitude for the elastic electron-proton scattering [1] is

$$
M_{e p \rightarrow e p}=\frac{1}{q^{2}}\left[e \overline { u } ( k _ { 2 } ) \gamma ^ { \mu } u ( k _ { 1 } ] \left[e \overline { U } \left(p_{2} \Gamma_{\mu}\left(p_{1}, p_{2}\right) U\left(p_{1}\right],\right.\right.\right.
$$

where $u$ and $U$ are the electron and nucleon Dirac spinors,

$$
\Gamma^{\mu}=F_{1}(t) \gamma^{\mu}+F_{2}(t) \frac{i \sigma^{\mu v} q_{v}}{2 m},
$$

where $m$ is the nucleon mass, $\kappa$ is the anomalous part of the magnetic moment and $t=-q^{2}=$ $-\left(p-p^{\prime}\right)^{2}$ is the square of the momentum transfer of the nucleon. The functions $F_{1}(t)$ and $F_{2}(t)$ are named the Dirac and Pauli form factors, which depend upon the nucleon structure. The normalization of the form factors [2] is given by

$$
F_{1}^{p}(t=0)=1, \quad F_{2}^{p}(t=0)=\kappa_{p}=1.793
$$

for the proton and

$$
F_{1}^{n}(t=0)=0, \quad F_{2}^{n}(t=0)=\kappa_{n}=-1.913
$$

\footnotetext{
*e-mail: selugin@ theor.jinr.ru
} 
for the neutron.

Two important combinations of the Dirac and Pauli form factors are the so-called Sachs form factors $[3,4]$. In the Breit frame the current is separated into the electric and magnetic contributions [5]

$$
\bar{u}\left(p^{\prime}, s^{\prime}\right) \Gamma^{\mu} u(p, s) \chi_{s^{\prime}}^{\dagger}\left(G_{E}(t)+\frac{i \vec{\sigma} \times \overrightarrow{q_{B}}}{2 m} G_{M}(t)\right) \chi_{s},
$$

where $\chi_{s}$ is the two-component of the Pauli spinor, $G_{E}(t)$ and $G_{M}(t)$ are the Sachs form factors given by

$$
\begin{aligned}
& G_{E}^{p / n}(t)=F_{1}^{p / n}(t)-\tau F_{2}^{p / n}(t), \\
& G_{M}^{p / n}(t)=F_{1}^{p / n}(t)+F_{2}^{p / n}(t),
\end{aligned}
$$

where $\tau=t /\left(4 M^{2}\right)$. Their three-dimensional Fourier transform provides the electric charge density and the magnetic current density distribution [4]. The form factors can be extracted from experimental data on the elastic electron-nucleon scattering by the Rosenbluth method [6] or from the polarization electron proton elastic scattering [7].

The radii of the particles are determined through the slope of the form factors at zero momentum transfer, and the squares of the Dirac radius $<r_{D}^{2}>$ and charge radius $<r_{E}^{2}>$ are determined as

$$
<r_{D}^{2}>=-\left.6 \frac{d F_{1}^{p / n}(t)}{d t}\right|_{t=0} ; \quad<r_{E}^{2}>=-\left.6 \frac{d F_{1}^{p / n}(t)}{d t}\right|_{t=0}+\frac{3}{2} \frac{k_{p / n}}{m_{p / n}^{2}},
$$

There are some problems in the description of the form factors at small momentum transfer. To obtain a derivative of the form factors at $t=0$, we must have the analytic form of the form factors. However, such analytic forms are obtained by fitting experimental data in a wide region of momentum transfer. Hence, the form of the analytic function can be determined in most part by the data at non-small momentum transfer. As a result, such a form at very small $t$ can not satisfy the experimental data in the region of very small $t$. For example, the latest experimental data [8] show a large difference from the standard dipole form of the electromagnetic form factors at $0.01<Q^{2}<0.016 \mathrm{GeV}^{2}$. If we take the standard dipole form of the electromagnetic form factors $\Lambda^{4} /\left(\Lambda^{2}-t\right)^{2}$ with $\Lambda^{2}=0.71 \mathrm{GeV}^{2}$, the analytic calculations give $\left\langle r_{D}^{2}>=0.76 \mathrm{fm}\right.$ and $\left\langle r_{E}^{2}>=0.88 \mathrm{fm}\right.$. It coincides with recent data for $\left\langle r_{E}^{2}\right\rangle=0.875(18) \mathrm{fm}$ [9] obtained from electronic hydrogen transition and experimental data on electron scattering [9]. Such parametrization takes into account only the experimental data for the charge proton form factor measured in the electron-proton scattering. There is a difference between the value of the proton radius and the determination of $\left\langle r_{E}^{2}\right\rangle$ in the muon-nucleon bound state, which gives $\left\langle r_{E}^{2}\right\rangle=0.84087$ (39) $f m[10,11]$. This discrepancy, known as the "proton radius puzzle", gave birth to a wide discussion and many approaches to explain this fact. But up to now the effect has no clear explanation.

The Parton distribution functions (PDF) are obtained in the deep inelastic scattering using the recent data at HERA and LHC. Beside this main point of the modern picture of the hadron structure, which depends only on the Bjorken longitudinal variable $x$, there were introduced a number of other more complicated parton distributions which can depend on a number of different variables. One of them is the Generelized parton distribution function (GPDs) (which depends on the Bjorken variable $x$, momentum transfer $t$ and skewnes parameter $\xi$ ) [13-15]. The conjunction between the momentum transfer and the impact parameter allow one to obtain the space parton distribution which has the probability conditions [12]. The connections 
between the deep-inelastic scattering, from which we can obtain the $x$-dependence of parton distributions, and the elastic electron-nucleon scattering, where the form factors of the nucleons are obtained, can be derived by using the sum rules [13-15]. The form factors, which are obtained in different reactions, can be calculated as the Mellin moments of GPDs. Using the electromagnetic (calculated as the zero Mellin moment of GPDs) and gravitomagnetic form factors (calculated as the first moment of GPDs) in the hadron scattering amplitude, one can obtain a quantitative description of the hadron elastic scattering in a wide region of energy and transfer momenta.

Many different forms of the $t$-dependence of GPDs were proposed. In the quark di-quark model [16] the form of GPDs consists of three parts - PDFs, function distribution and Reggelike function. In other works (see e.g. [17]), the description of the $t$-dependence of GPDs was developed in a more complicated representation using the polynomial forms with respect to $x$.

\section{Momentum transfer dependence of GPDs}

To obtain the true form of the proton and neutron form factors, it is important to have the true form of the momentum transfer dependence of GPDs. Let us choose the $t$-dependence of GPDs in a simple form $\mathcal{H}^{q}(x, t)=q(x) \exp \left[a_{+} f(x) t\right]$, with $f(x)=(1-x)^{2} / x^{\beta}$ [18]. The isotopic invariance can be used to relate the proton and neutron GPDs, hence we have the same parameters for the proton and neutron GPDs.

The complex analysis of the corresponding description of the electromagnetic form factors of the proton and neutron by different PDF sets (24 cases) was carried out in [19]. These PDFs include the leading order (LO), next to leading order (NLO) and next-next to leading order (NNLO) determination of the parton distribution functions. They used different forms of the $x$ dependence of PDFs. We slightly complicated the form of GPDs in comparison with the equation used in [18], but it is the simplest one as compared to other works (for example [20]).

The hadron form factors will be obtained by integration over $x$ in the whole range of $x$ $0-1$. Hence, the obtained form factors will be dependent on the $x$-dependence of the forms of PDF at the ends of the integration region. The Collaborations determined the PDF sets from the inelastic processes only in some region of $x$, which is only approximated to $x=0$ and $x=1$. Some PDFs have the polynomial form of $x$ with different power. Some others have the exponential dependence of $x$. As a result, the behavior of PDFs, when $x \rightarrow 0$ or $x \rightarrow 1$, can impact the form of the calculated form factors.

On the basis of our GPDs with, for example the PDFs ABM12 [21], we calculated the hadron form factors by numerical integration and then by fitting these integral results by the standard dipole form with some additional parameters

$$
F_{1}(t)=\left(4 m_{p}-\mu t\right) /\left(4 m_{p}-t\right) \tilde{G}_{d}(t)
$$

with

$$
\tilde{G}_{d}(t)=1 /\left(1+q / a_{1}+q^{2} / a_{2}^{2}+q^{3} / a_{3}^{3}\right)^{2}
$$

which is slightly different from the standard dipole form on two additional terms with small sizes of coefficients. The matter form factor

$$
A(t)=\int_{0}^{1} x d x\left[q_{u}(x) e^{2 \alpha_{H} f(x)_{u} / t}+q_{d}(x) e^{2 \alpha_{H} f_{d}(x) / t}\right]
$$

is fitted by the simple dipole form $A(t)=\Lambda^{4} /\left(\Lambda^{2}-t\right)^{2}$. These form factors will be used in our model of the proton-proton and proton-antiproton elastic scattering and further in one of the vertices of the pion-nucleon elastic scattering. 
To check the momentum dependence of the spin-dependent part of GPDs $E_{u, d}(x, \xi=$ $0, t)$, we can calculate the magnetic transition form factor [22], which is determined by the difference of $E_{u}(x, \xi=0, t)$ and $E_{d}(x, \xi=0, t)$. For the magnetic $N \rightarrow \Delta$ transition form factor $G_{M}^{*}(t)$, in the large $N_{c}$ limit, the relevant $G P D_{N \Delta}$ can be expressed in terms of the isovector GPD yielding the sum rules [23] The experimental data exist up to $-t=8 \mathrm{GeV}^{2}$ and our results show a sufficiently good coincidence with experimental data. It is confirmed that the form of the momentum transfer dependence of $E(x, \xi, t)$ determined in our model is correct. The moments of the GPDs with inverse power of $x$ gives us the Compton form factors. The results of our calculations of the Compton form factors coincide well with the existing experimental data. For $H^{q}(x, t), E^{q}(x, t)$ we used the PDFs obtained from the works [24] with the parameters obtained in our fitting procedure of the description of the proton and neutron electromagnetic form factors in [19].

\section{Electromagnetic and gravitomagnetic form factors and the elastic nucleon-nucleon scattering}

Both hadron electromagnetic and gravitomagnetic form factors were used in the framework of the high energy generalized structure (HEGS) model of the elastic nucleon-nucleon scattering. This allowed us to build the model with a minimum number of fitting parameters [25-28].

The Born term of the elastic hadron amplitude can now be written as

$$
\begin{aligned}
F_{h}^{\text {Born }}(s, t)=\quad & h_{1} G^{2}(t) F_{a}(s, t)\left(1+r_{1} / \hat{s}^{0.5}\right)+h_{2} A^{2}(t) F_{b}(s, t) \\
& \pm h_{\text {odd }} A^{2}(t) F_{b}(s, t)\left(1+r_{2} / \hat{s}^{0.5}\right),
\end{aligned}
$$

where both (electromagnetic and gravitomagnetic) form factors are used. The parameters are determined in [26]. The model is very simple from the viewpoint of the number of fitting parameters and functions. There are no any artificial functions or any cuts which bound the separate parts of the amplitude by some region of momentum transfer. In the framework of the model the description of experimental data was obtained simultaniously at the large momentum transfer and in the Coulomb-hadron region in the energy region from $\sqrt{s}=9$ $\mathrm{GeV}$ up to LHC energies with taking into account the Coulomb-nuclear phase $[29,30]$. The model gives a very good quantitative description of recent experimental data at $\sqrt{s}=13 \mathrm{TeV}$ [31].

\section{Nucleon gravitomagnetic radii}

A good description of the various form factors and the elastic scattering of the hadrons gives a good support to our determination of the momentum transfer dependence of GPDs. Based on this determination of GPDs, let us calculate the gravitomagnetic radius of the nucleon using the integral representation of the form factor and make the numerical differentiation over $t$ at $t \rightarrow 0$. This method allows us to obtain a concrete form of the form factor by fitting the result of the integration of the GPDs over $x$. As a result, the gravitomagnetic radius is determined as

$$
<r_{A}^{2}>=-\left.\frac{6}{A(0)} \frac{d A(t)}{d t}\right|_{t=0}
$$

hence the numerical derivative will be

$$
<r_{A}^{2}>=-\frac{6}{A(0)} \frac{A\left(t_{1}\right)-A\left(t_{1}+\Delta t\right.}{\Delta t} \mid
$$


Table 1. The gravitation radius of the nucleon

\begin{tabular}{|c|c|c|c|c|c|c|}
\hline \multirow{3}{*}{$\begin{array}{l}\text { Model } \\
\text { of PDFs }\end{array}$} & \multicolumn{3}{|c|}{$\left(<r_{A}^{2}\right)>^{1 / 2}$ basic variant } & \multicolumn{3}{|c|}{$\left(<r_{A}^{2}\right)>^{1 / 2}$ basic +4 parameters } \\
\hline & \multirow[t]{2}{*}{$\begin{array}{c}-t_{1} \\
10^{-5} \\
\Delta t=10^{-5} \\
\mathrm{GeV}^{2}\end{array}$} & $\begin{array}{r}-t_{1} \\
10^{-3} \\
\Delta t\end{array}$ & $\begin{array}{c}-t_{1} \\
410^{-2} \\
10^{-3}\end{array}$ & \multirow[t]{2}{*}{$\begin{array}{c}-t_{1} \\
0.001 \\
\Delta t=10^{-5} \\
\mathrm{GeV}^{2}\end{array}$} & $\begin{array}{c}-t_{1} \\
0.001\end{array}$ & $\begin{array}{c}-t_{1} \\
0.02 \\
=10^{-3}\end{array}$ \\
\hline & & $\mathrm{GeV}^{2}$ & $\mathrm{GeV}^{2}$ & & $\mathrm{GeV}^{2}$ & $\mathrm{GeV}^{2}$ \\
\hline ABKM09 & 0.525 & 0.525 & 0.505 & 0.536 & 0.535 & $0.522[\mathrm{fm}]$ \\
\hline JR08a & 0.546 & 0.545 & 0.547 & 0.566 & 0.565 & $0.543[\mathrm{fm}]$ \\
\hline JR08b & 0.526 & 0.525 & 0.507 & 0.547 & 0.547 & $0.527[\mathrm{fm}]$ \\
\hline ABM12 & 0.524 & 0.524 & 0.505 & 0.543 & 0.543 & $0.523[\mathrm{fm}]$ \\
\hline KKT12a & 0.525 & 0.524 & 0.504 & 0.525 & 0.525 & $0.507[\mathrm{fm}]$ \\
\hline KKT12b & 0.519 & 0.519 & 0.521 & 0.498 & 0.518 & $0.501[\mathrm{fm}]$ \\
\hline GJR07d & 0.523 & 0.523 & 0.505 & 0.531 & 0.531 & $0.515[\mathrm{fm}]$ \\
\hline GJR07b & 0.532 & 0.531 & 0.512 & 0.546 & 0.544 & $0.526[\mathrm{fm}]$ \\
\hline GJR07a & 0.506 & 0.506 & 0.498 & 0.521 & 0.520 & $0.503[\mathrm{fm}]$ \\
\hline GJR07c & 0.510 & 0.510 & 0.501 & 0.519 & 0.519 & $0.502[\mathrm{fm}]$ \\
\hline
\end{tabular}

where $A(t)=\int_{0}^{1} x 3\left(q_{u}(x)+q_{d}(x)\right) e^{-\alpha t f(x)} d x$

The GPDs will be taken with various forms of the PDFs obtained by different Collaborations (see our paper [19]). To compare the $t$ dependence of the starting point of the differentiation of the results, we take 3 variants

$$
\begin{aligned}
& 1)-t_{1}=1.10^{-3} \text { and } \Delta t_{1}=1.10^{-3} ; \\
& 2)-t_{1}=1.10^{-2} \text { and } \Delta t_{1}=1.10^{-3} ; \\
& 3)-t_{1}=4.10^{-2} \text { and } \Delta t_{1}=1.10^{-3} ;
\end{aligned}
$$

To examine the dependence of the results on our model parametrization of GPDs, we made the calculation for the simplest basic variant with minimum parameters and a more complicated variant with additional 4 parameters. It was shown in [19] that the final result of the fitting of GPDs on the basis of practically all experimental data on the electromagnetic form factors of the proton and neutron, weakly depended on adding the supplementary fitting parameters but some others had a heavy dependence. We put the GPDs of the first class in the upper rows of Table 1. It is clear from the comparison of the variants 1) and 2) (see Table 1) that there is no difference between the results obtained at $-t=10^{-5} \mathrm{GeV}^{2}$ and $-t=10^{-3}$ $\mathrm{GeV}^{2}$. However, already at $-t=4 \cdot 10^{-2} \mathrm{GeV}^{2}$ the results have significant differences. Hence, if we make the fitting procedure to obtain the analytic form of the form factors, the region of a very small momentum transfer has to be leading.

Note that the "basic" and the "basic + 4 parameters" variants can lead to the same different results for the PDFs of different Collaborations. For example, the PDFs of (KKT12a [24]) give the radius which is practically independent of additional free parameters. Hence, this result can be considered as most stable and probable. Note that this size of the radius practically coincides with the arithmetic means of all radii (in the first column), which is equal to $\left(<r_{A}^{\overline{2}}\right)>^{1 / 2}=0.524 \mathrm{fm}$. 
Table 2. The electromagnetic radii of the nucleon

\begin{tabular}{|c|c|c|c|c|c|c|c|}
\hline \multirow[t]{2}{*}{$\mathrm{N}$} & \multirow[t]{2}{*}{ Model } & \multirow[t]{2}{*}{ Refer. } & \multicolumn{2}{|c|}{$<r_{D}>$} & \multicolumn{2}{|c|}{$<r_{G}>$} & \multirow[t]{2}{*}{ Order(Q) } \\
\hline & & & basic & $+4_{\text {par }}$ & basic & $+4_{\text {par }}$ & \\
\hline 1 & ABKM09 & [33] & 0.787 & 0.787 & 0.859 & $0.859[\mathrm{fm}]$ & NNLO (3.) \\
\hline $2 \mathrm{a}$ & JR08a & [32] & 0.808 & 0.793 & 0.879 & $0.865[\mathrm{fm}]$ & NNLO $(0.55)$ \\
\hline $2 b$ & JR08b & [32] & 0.789 & 0.793 & 0.861 & $0.865[\mathrm{fm}]$ & NNLO (2.) \\
\hline 3 & ABM12 & [21] & 0.778 & 0.787 & 0.851 & $0.859[\mathrm{fm}]$ & NNLO (0.9) \\
\hline $4 a$ & KKT12a & [24] & 0.788 & 0.789 & 0.860 & $0.861[\mathrm{fm}]$ & NLO (4.) \\
\hline $4 b$ & KKT12b & [24] & 0.780 & 0.796 & 0.853 & $0.867[\mathrm{fm}]$ & NLO (4.) \\
\hline $6 a$ & GJR07d & [34] & 0.735 & 0.781 & 0.811 & $0.853[\mathrm{fm}]$ & NLO (0.3) \\
\hline $5 b$ & GJR07b & [34] & 0.788 & 0.781 & 0.860 & $0.853[\mathrm{fm}]$ & $\operatorname{NLO}(0.3)$ \\
\hline $5 c$ & GJR07a & [34] & 0.766 & 0.775 & 0.840 & $0.848[\mathrm{fm}]$ & LO (0.3) \\
\hline $5 d$ & GJR07c & [34] & 0.748 & 0.775 & 0.824 & $0.848[\mathrm{fm}]$ & NLO (0.3) \\
\hline
\end{tabular}

\section{Nucleon electromagnetic radii}

Now let us calculate the Dirac radius of the proton $\left\langle r_{D}^{2}>\right.$ and the charge radius of the proton $<r_{E}^{2}>$ using our momentum transfer dependence of GPDs. We used the same procedure as for our calculations of the matter radius. As a result, the Dirac radius is determined from the zero Mellin moment of GPDs

$$
<r_{D}^{2}>=-\left.\frac{6}{F(0)} \frac{d F(t)}{d t}\right|_{t=0}
$$

hence the differentiation will be

$$
<r_{D}^{2}>=-\left.\frac{6}{F(0)} \frac{F\left(t_{1}\right)-F\left(t_{1}+\Delta t\right)}{\Delta t}\right|_{t \rightarrow 0} ;
$$

where

$$
F(t)=\int_{0}^{1}\left(e_{u} q_{u}(x)+e_{d} q_{d}(x)\right) e^{-\alpha t f(x)} d x .
$$

Now we consider only the first variant with $t_{1}=10^{-5} \mathrm{GeV}^{2}$ and $\Delta t=10^{-5} \mathrm{GeV}^{2}$.

The results are presented in Table 2. Again, we see that a variant of PDFs gives the result which weekly depends on the number of free parameters. If the arithmetic mean of $\left(<r_{e}{ }^{2}>^{1 / 2}\right)$ is calculated, we find that the charge radius $\left(<r_{e}{ }^{-}>^{1 / 2}\right)=0.850 \mathrm{fm}$ for the basic variant and $\left(\left(<r_{e} \overline{2}>^{1 / 2}=0.860\right) \mathrm{fm}\right.$ for the variant with additional four fitting parameters. These values are between the sizes of the charge proton radii determined in the electron and muon experiments. In some sense, this removes the discrepancy between these two methods. But, of course, our errors, especially which come from the $x$-dependence of different PDFs at $x \rightarrow 0$ and $x \rightarrow 1$, are large, and this requires further deep analysis.

\section{Conclusion}

The structure of the hadron represented by the Generelazed parton distribution functions is the main part in the amplitudes of different reactions. The Mellin moments of GPDs allow 
us to calculate different form factors from the same analytic functions. The parameters of the phenomenological form of GPDs can be obtained from the analysis of the experimental data of the proton and neutron electromagnetic form factors simultaneously. Our determination of the momentum transfer dependence of GPDs of hadrons allows us to obtain good quantitative descriptions of different form factors, including the Compton, electromagnetic, transition and gravitomagnetic form factors simultaneously. Hence, our calculations of the nucleons radii are based on the use of the maximum number of experimental data.

Our method of the calculation of the radius from the numerical derivation of the integral of GPDs over variable $x$ does not require any fitting of separate hadron form factors. Most significant uncertainty in the determination of the nucleon radii comes from the indeterminacy of the form of the parton distribution function which has the phenomenological origin with the parameters determined from the analysis of different deep inelastic reactions. Our calculations show the low values of the charge radius of the proton compared to the standard determination of the dipole form of the proton form factor. In some sense, this removes the contradictions with the size of the charge radius of the proton obtained in the muon experiment. Of course, the uncertainty which comes from different forms of the PDFs is large now, and this requires further analysis.

Acknowledgments The authors would like to thank J.-R. Cudell and O.V. Teryaev for fruitful discussion of some questions considered in the paper.

\section{References}

[1] E.L. Lomon, S. Pacetty Phys.Rev. D 86, 039901 (2012).

[2] I.A. Qattan and J. Arrington, Phys.Rev. C 86, 065210 ( 2012).

[3] F.J. Ernst, R.G. Sachs, and K.C. Wali Phys. Rev. 119, 1105 (1960).

[4] R.G. Sachs, Phys.Rev. 126, 2256 (1962).

[5] J.J. Kelly, Phys.Rev. C 66, 065203 (2002).

[6] M.N. Rosenbluth, Phys.Rev. 79615 (1950).

[7] A.I. Akhiezer and M.P. Rekalo Sov.J.Pat.Nucl. 3277 (1974).

[8] M. Mihovilovic et al. , Phys.Lett., bf B 771194 (2017).

[9] P.J. Mohr, D.B. Newell, and B.N. Taylor, Rev.Mod.Phys. 88, 035009 (2016).

[10] R. Pohl et al., Nature 466417 (2010)

[11] M. Mihovilovic et al., Phys.Lett,, bf 771194 (2017).

[12] M. Burkardt and B. Pasquini, EPJA Special Issue on "3D Structure of the Nucleon"; EPJ,

[13] D. Muller et al., Fortsch. Phys. 42, (1994) 101;

[14] X.D. Ji, Phys. Lett. 78 , (1997) 610; Phys. Rev D 55 (1997) 7114;

[15] Radyushkin, A.V., Phys. Rev. D 56, 5524 (1997).

[16] G.R. Goldstein, J.O. Hernandez, S. Liuti, Phys.Rev. D84 034007 (2011).

[17] M.Diehl et al., Eur.Phys. J. C 39 (2005) 1.

[18] O. Selyugin, O. Teryaev, Phys. Rev. D 79033003 (2009);

[19] O.V. Selyugin, Phys. Rev. D 89093007 (2014) .

[20] M. Diehl and P. Kroll, Eur.Phys.J. C73 2397 (2013).

[21] S. Alekhin, J. Blu"mlein, and S. Moch, Phys.Rev. D86, 054009 (2012).

[22] O.V. Selyugin, in Procceding of XXVII Int. workshop "Spin in high energy physics", Dubna (2017); J.Phys.Conf.Ser. 938 (2017). 
[23] M. Guidal, M. V. Polyakov, A. V. Radyushkin, M. Vanderhaeghen, PhysRevD. D72 054013 (2004).

[24] H. Khanpour et al., arXiv:1205.5194

[25] O.V. Selyugin, Eur.Phys.J. C72, 2073 (2012).

[26] O.V. Selyugin, Phys. Rev. D 91113003 (2015).

[27] O. V. Selyugin, Nucl.Phys. A 90354 (2013).

[28] O.V. Selyugin, Nucl.Phys. A 959116 (2017).

[29] O.V. Selyugin, Mod. Phys. Lett. A9 1207 (1994).

[30] O. V. Selyugin, Phys. Rev. D 60 (1999) 074028

[31] O. V. Selyugin, Phys.Lett. B 7971134870 (2019).

[32] M. Gluck, Phys.Rev., D79 , 074023 (2009).

[33] S. Alekhin et al., Phys.Rev. D81, 014032 (2010).

[34] M. Gluck, P. James-Delgado, E. Reya, Eur.Phys.J., C53 355 (2008). 